\title{
Anterior versus posterior approach laparoscopic radical cystectomy: a retrospective analysis
}

Shiyu Tong ${ }^{\dagger}$, Zhenyu Yang ${ }^{\dagger}$, Xiongbin Zu, Yuan Li, Wei He, Yangle Li, Xiheng Hu, Cheng Zhao, Minfeng Chen ${ }^{*}$ (D) and Lin Qi*

\begin{abstract}
Objective: To investigate the mortality, operation time, cystectomy time, and complications of anterior approach laparoscopic radical cystectomy (ALRC) in Asian males in comparison with posterior approach laparoscopic radical cystectomy (PLRC).
\end{abstract}

Materials and methods: One hundred forty-seven male patients with bladder cancer (cT2-3NxM0) in our hospital from May 2011 to January 2018 having undergone laparoscopic radical cystectomy were studied, including 68 patients in PLRC group and 79 patients in ALRC group. Baseline patient characteristics, operative and postoperative characteristics, and postoperative complications were retrospectively collected and analyzed between the two groups.

Results: Patients in these two groups exhibited similar baseline characteristics $(p>0.05)$. Compared with PLRC group, ALRC group required similar operation time $(317.3 \pm 40.9$ vs $321.9 \pm 37.5)$ and cystectomy time $(64.8 \pm 8.7$ vs $65.6 \pm 14$. 0). The ALRC group required less cystectomy time $(67.8 \pm 10.1$ vs $77.4 \pm 14.9)$ when patients' BMI > 24 or patients had large total tumor and blood clot volume $\left(>160 \mathrm{~cm}^{3}\right.$ ). Also, estimated blood loss (EBL) of ALRC group was significantly less than that of PLRC group (477.8 \pm 97.4 vs $550.4 \pm 99.9)$. There existed no significant differences between the PLRC and ALRC groups in postoperative characteristics and complications.

Conclusion: This study revealed that ALRC required less cystectomy time for patients with higher BMI and larger tumor, suggesting less blood loss and similar perioperative complications. ALRC is recommend for male patients, of which BMI $>24$ or total tumor and blood clot volume $>160 \mathrm{~cm}^{3}$.

Keywords: Bladder cancer, Bladder suspension, Anterior approach laparoscopic radical cystectomy, Complication

\section{Introduction}

Bladder cancer $(\mathrm{BCa})$, characterized by high risk of recurrence and mortality, is a serious health risk worldwide, with an estimated 430,000 new cases in 2012 [1]. BCa is common in elder individuals and strongly associated with smoking exposure $[2,3]$. The incidence of $\mathrm{BCa}$ has been rising in China with the population aging, and $\mathrm{BCa}$ ranks the first in the urinary malignant tumor with an incidence of $69.7 / 100,000$ [4]. Accordingly, the treatment for bladder cancer is of huge importance.

\footnotetext{
* Correspondence: chenminfeng1999@163.com; linqi1112@yeah.net 'Shiyu Tong and Zhenyu Yang contributed equally to this work. Department of Urology, Xiangya Hospital, Central South University, 87 Xiangya Rd, Changsha 410008, Hunan, People's Republic of China
}

Radical cystectomy $(\mathrm{RC})$ is the standard management of non-metastatic, muscle-invasive $\mathrm{BCa}$ [5]. Though open radical cystectomy (ORC) is a universally accepted gold standard for treating muscle-invasive, organ-confined $\mathrm{BCa}$, minimally invasive surgical techniques, such as laparoscopic radical cystectomy (LRC) and robot-assisted laparoscopic radical cystectomy (RALRC), have been rapidly accepted for treating BCa. Compared with ORC, these new techniques with small incisions are considered to cause less blood loss and shorter hospital stay, while yielding equivalent oncologic and functional outcomes [6-8]. However, LRC has not been widespread because of the technical challenges of the procedure. The process of LRC is long and complicated, requiring detailed knowledge of

(c) The Author(s). 2019 Open Access This article is distributed under the terms of the Creative Commons Attribution 4.0 International License (http://creativecommons.org/licenses/by/4.0/), which permits unrestricted use, distribution, and 
pelvic anatomy. LRC is really challenging for male patients with higher BMI and larger tumors, even for an experienced surgical team. As the working space in the narrow pelvis would be smaller, laparoscopic visualization would be limited.

The LRC techniques for male fat patients or male patients with larger tumor and blood clot have been rarely studied. To make better laparoscopic visualization and bigger working space, posterior approach laparoscopic radical cystectomy (PLRC) was modified in 2011, and bladder suspension was performed during the laparoscopic radical cystectomy. To compare PLRC with anterior approach laparoscopic radical cystectomy (ALRC), 147 male patients who underwent PLRC or ALRC in our hospital from May 2011 to January 2018 in this study were retrospectively analyzed, and our experience of ALRC was reported.

\section{Materials and methods Patients}

This study was approved by the ethics board of Xiangya Hospital, Central South University, in Changsha, China. All the participants were informed that their clinical information may be applied in later clinical study when they entered hospital. Also, their written informed consents were given. Between May 2011 and January 2018, there were 157 male patients in our hospital, with high-grade bladder urothelial carcinoma, of which 72 and 85 patients are in the PLRC and ALRC group respectively; we failed to follow up 4/72 patients in PLRC group and 6/85 patients in ALRC group. Thus, these patients were excluded, and the medical records of the 147 patients retrospectively were collected and analyzed from our bladder cancer database. We made the technique rotation from month to month. All patients have high-grade bladder urothelial carcinoma $\left(\mathrm{cT}_{2-3} \mathrm{~N}_{\mathrm{X}} \mathrm{M}_{0}\right)$ in line with TMN classification of the Union for International Cancer Control [9]. All patients had primary tumors, and none of the patients had received bladder surgeries, radiotherapy, or chemotherapy before.

Patients were evaluated in accordance with the American Society of Anesthesiologists (ASA) physical status classification system. Each patient underwent preoperative examination including routine laboratory test, echocardiography, chest radiography, lung function test, computerized tomography, or magnetic resonance imaging of urinary system. Common comorbidities were recorded, including diabetes mellitus, coronary artery disease, hypertension, chronic obstructive pulmonary disease, and other chronic diseases. Patients began semi-liquid diet $48 \mathrm{~h}$ before surgery, liquid diet $24 \mathrm{~h}$ before surgery, and underwent bowel preparation with enema and oral sodium phosphates solution $12 \mathrm{~h}$ before surgery. All patients got broad-spectrum systemic antibiotic intravenously during the induction of anesthesia. Same laparoscopic instruments and devices from KARL STORZ were used in both groups. All the operations and perioperative management were performed by the same experienced surgical team. Three surgeons (MC, LQ, and XZ) performed the operations. MC performed 30 and 35, LQ performed 22 and 24, and XZ performed 16 and 20 in PLRC and ALRC respectively. All the surgeons are very experienced and perform more than 25 cases of laparoscopic radical cystectomy annually. Follow-up studies were conducted with ultrasonography and CT scan at 3 and 9 months postoperatively.

\section{Parameters and endpoint}

The baseline patient characteristics included age, BMI, hemoglobin $(\mathrm{Hb})$, total tumor and blot clot volume, comorbid conditions, and ASA score. Total tumor and blot clot volume were measured with CT scan. Operative characteristics included operation time, cystectomy time, estimated blood loss (EBL), and transfusion needed, while postoperative characteristics included $\mathrm{Hb}$, time to liquid intake, time to exsufflation, and hospital stay after surgery. Operation time was defined as anesthesia from the beginning to the end, and cystectomy time was defined as procedure from starting mobilization to bladder removed completely. Intraoperative complication includes rectal injury and external iliac vein injury. Complications that occurred within 90 days after surgery were considered early complications, which included ileus, deep vein thrombosis, pyelonephritis, infection, obturator nerve paresis, and wound dehiscence [10]. The complication occurring within 90 days or later after surgery were defined as late complications, including ileus, ureteral stricture, and incisional hernia [10].

\section{Statistical analysis}

Chi-square test and Student's $t$ test were performed to compare categorical and parametric data respectively between the two groups. Parametric data is denoted as mean $\pm \mathrm{SD}$, and categorical data is presented as frequency (\%). Linear regression analysis was conducted to assess the relationship between cystectomy time and total tumor and blood clot volume. Significant differences were considered when $p<0.05$. Statistical analysis was conducted with the Statistical Package for Social Scientists, version 22.0 (IBM Inc).

\section{Laparoscopic radical cystectomy}

The basic laparoscopic radical cystectomy procedures were performed according to Campbell-Walsh Urology [11], A five-port fan-shaped trans-peritoneal approach was employed for LRC. The camera port was placed just below the umbilicus. The residual four ports were placed under endoscopic control after the establishment 
of the pneumoperitoneum. A dorsal supine position with a $20-25^{\circ}$ Trendelenburg position was used to keep the bowel from the pelvis during surgery. Surgery was performed by visualizing the pelvis and releasing adhesions of the sigmoid colon to find the anatomical landmarks in the pelvis, such as obliterated umbilical arteries, obliterated urachus, spermatic cord, and vas deferens. The standard lymphadenectomy was performed in all cases [12]. After laparoscopic cystectomy, urinary diversion, bricker operation, or orthotopic neobladder, was conducted according to patients' preferences.

\section{Posterior and anterior approach laparoscopic radical cystectomy}

The posterior approach laparoscopic radical cystectomy is a conventional LRC, the posterior plane was developed first, and the whole operation procedure was performed according to Campbell-Walsh Urology [11]. The anterior approach laparoscopic radical cystectomy was performed as follows. The anterior plane was first developed distally towards the prostate. Both puboprostatic ligaments were mobilized and dissected, and the Santorini venous plexus was ligated. Subsequently, the bladder was suspended towards the abdominal wall with a 2-0 synthetic non-absorbable suture (Fig. 1a). The laparoscopy observation showed that nearly half of the needle was punctured into the abdominal, $3 \mathrm{~cm}$ above the pubic symphysis, and about $2 \mathrm{~cm}$ right to the midline (Fig. 1b). The needle was punctured through the right side of bladder wall, then intermittently through the posterior and left side, puncturing out to the symmetrical position of the abdominal wall (Fig. 1c-f). The needle should be avoided from penetrating the bladder wall, and the suture should be limited in the superficial peritoneum. After puncturing out the abdominal wall (Fig. 1g), the end of this suture was knotted with tension. The vesicorectal fossa could extend to the fullest after bladder suspension was completed (Fig. 1h). After bladder suspension, the posterior plane was developed. Next, the procedures of handling the posterior and lateral planes were the same as those of PLRC. After the posterior and lateral bladder attachments were released, the bladder was released from the anterior abdominal wall. The attachments of the prostatic apex to the pelvic floor were released, and the urethral catheter was removed. After the dissection of Santorini venous plexus, the urethra was dissected.

\section{Results}

\section{Baseline patient characteristics}

In general, 147 patients were recruited here, including 68 patients who underwent PLRC and 79 patients proceeded with ALRC. Patients in the PLRC group and ALRC group had comparable baseline characteristics,

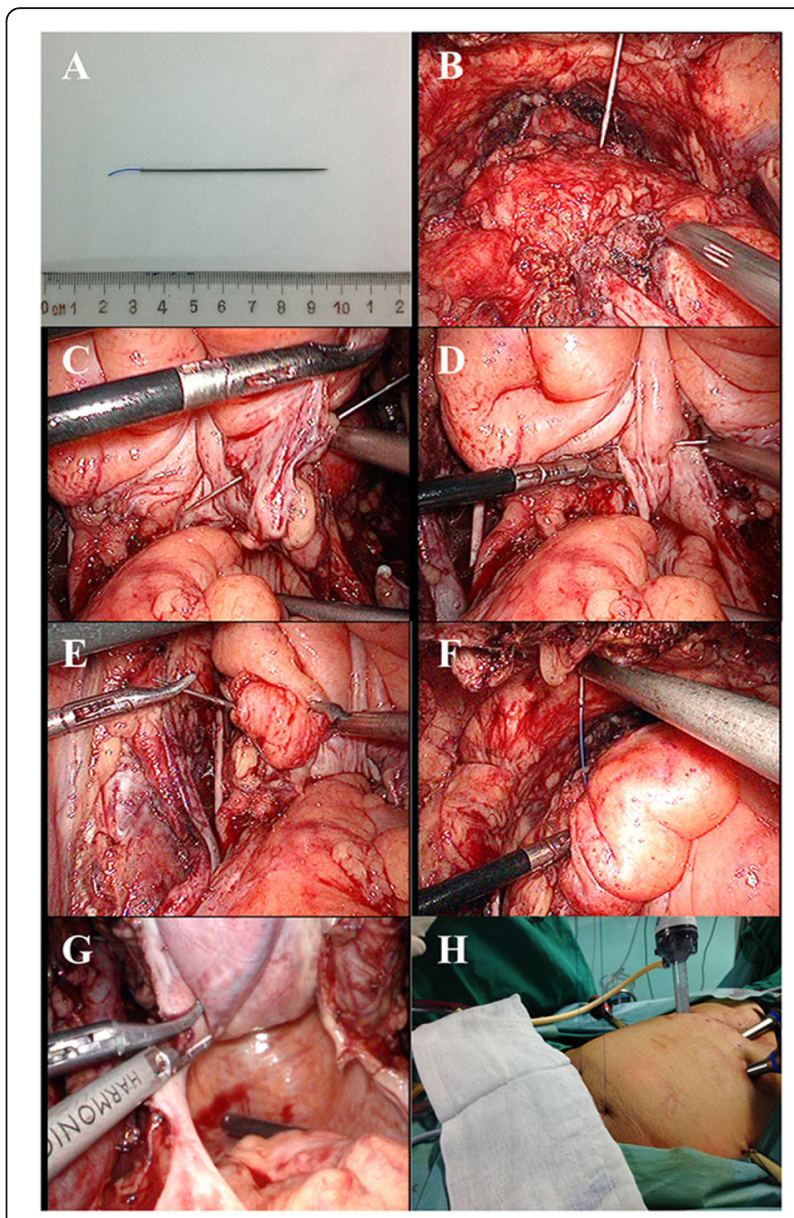

Fig. 1 Bladder suspension technique. a 2-0 synthetic non-absorbable (Polyamid 6, Ethilon*II) suture and needle. $\mathbf{b}$ The needle was puncturing into the abdomen. $\mathbf{c}$ The needle was puncturing through the right side bladder wall. $\mathbf{d}$ The needle was puncturing through the lower posterior bladder wall. e The needle was puncturing through the left side bladder wall. $\mathbf{f}$ The needle was puncturing through the abdominal wall out of abdomen. $\mathbf{g}$ The extended space of vesicorectal fossa with the help of bladder suspension. $\mathbf{h}$ View of the suture outside before knotting

including age, BMI, $\mathrm{Hb}$, total tumor and blood clot volume, comorbid conditions, and ASA score (Table 1).

\section{Operative and postoperative characteristics}

One patient in the PLRC group was transferred to open surgery, and no patients in the ALRC groups were transferred to open surgery. Operative and postoperative characteristics of the two groups are shown in Table 2. Estimated blood loss (EBL) of the ALRC group was significantly lower than that of the PLRC group $(477.8 \pm 97.4$ vs $550.4 \pm 99.9)$. Also, less patients in ALRC group needed transfusion (Table 2). The $\mathrm{Hb}$ after surgery in ALRC group was a little higher than the PLRC group $(102.1 \pm 11.6$ vs $98.1 \pm 12.5)$. There were no significant differences between the two groups in operation time, cystectomy time, time to liquid intake, time to exsufflation, and hospital stay after surgery. 
Table 1 Baseline patient characteristics

\begin{tabular}{|c|c|c|c|c|}
\hline Characteristics & $\operatorname{PLRC}(n=68)$ & $\operatorname{ALRC}(n=79)$ & $x^{2} / t$ & $p$ value \\
\hline Age, median (range) & $57(44-81)$ & $59(47-80)$ & $0.629^{*}$ & 0.531 \\
\hline $\mathrm{BMI}\left(\mathrm{kg} / \mathrm{m}^{2}\right)$ & $21.7 \pm 3.1$ & $22.3 \pm 3.3$ & $0.333^{*}$ & 0.730 \\
\hline $\mathrm{Hb}(\mathrm{g} / \mathrm{L})$ & $121.2 \pm 11.7$ & $120.6 \pm 10.5$ & $0.330^{*}$ & 0.742 \\
\hline Total tumor and blood clot volume & $179.0 \pm 72.2$ & $186.0 \pm 66.8$ & $0.697^{*}$ & 0.487 \\
\hline \multicolumn{5}{|l|}{ Comorbid conditions } \\
\hline Diabetes mellitus & $15(22.1 \%)$ & $20(25.3 \%)$ & 0.214 & 0.644 \\
\hline Hypertension & $20(29.4 \%)$ & $22(27.8 \%)$ & 0.044 & 0.834 \\
\hline Coronary heart disease & $5(7.4 \%)$ & $4(5.1 \%)$ & 0.333 & 0.564 \\
\hline COPD & $3(4.4 \%)$ & $4(5.1 \%)$ & 0.034 & 0.853 \\
\hline Other chronic diseases & $3(4.4 \%)$ & $2(2.5 \%)$ & 0.393 & 0.531 \\
\hline \multicolumn{5}{|l|}{ ASA score } \\
\hline 2 & $52(76.4 \%)$ & $60(75.9 \%)$ & 0.083 & 0.773 \\
\hline 3 & 16 (23.6\%) & 19 (24.1\%) & & \\
\hline
\end{tabular}

${ }^{*} t$ value

When patients were sub-grouped based on BMI, cystectomy time was different between sub-groups though the operation time $(317.3 \pm 40.9$ vs $321.9 \pm$ $37.5)$ and cystectomy time $(64.8 \pm 8.7$ vs $65.6 \pm 14.0)$ were similar between the two groups. When BMI was less than 18.5, the PLRC group took less cystectomy time compared with the ALRC $(52.5 \pm 6.5$ vs $60.3 \pm$ 6.2). When BMI was ranged from 18.5 to 24 , cystectomy time was similar between the two groups. It took longer cystectomy time for the PLRC group when BMI was greater than $24(77.4 \pm 14.9$ vs $67.8 \pm$ 10.1) (Table 3). Liner regression analysis was conducted to access the relationship between cystectomy time and total tumor and blood clot volume. In both PLRC $\left(y=0.1787 x+34.66, r^{2}=0.8225, p<0.0001\right)$ and ALRC $\left(y=0.0851 x+49.29, r^{2}=0.4459, p<0.0001\right)$, cystectomy time were correlated significantly positively with total tumor and blood clot volume. Both PLRC and ALRC required similar cystectomy time when total tumor and blood clot volume was about $160 \mathrm{~cm}^{3}$. Compared with ALRC, PLRC required less cystectomy time and operation time when total tumor and blood clot volume was less than $160 \mathrm{~cm}^{3}$, and PLRC required more cystectomy time and operation time when total tumor and blood clot volume was larger than $160 \mathrm{~cm}^{3}$ (Table 2, Fig. 2).

\section{Intraoperative and postoperative complications}

There were no deaths in the two groups during the perioperative period. Intraoperative and postoperative complications of the two groups are listed in Table 4. Two patients underwent rectal injury and another patient got external iliac vein injury in the PLRC group during surgery, and one patient underwent external iliac vein injury in the ALRC group. Ileus was

Table 2 Operative and postoperative characteristics

\begin{tabular}{|c|c|c|c|c|}
\hline Characteristics & $\operatorname{PLRC}(n=68)$ & $\operatorname{ALRC}(n=79)$ & $x^{2} / t$ & $p$ value \\
\hline Operation time (min) & $321.9 \pm 37.5$ & $317.3 \pm 40.9$ & $0.702^{*}$ & 0.484 \\
\hline $\begin{array}{l}\text { Operation time } \\
\left(\mathrm{min} \text {, volume }>160 \mathrm{~cm}^{3}\right)\end{array}$ & $\begin{array}{l}340.8 \pm 25.2 \\
(n=37)\end{array}$ & $\begin{array}{l}320.7 \pm 42.6 \\
(n=51)\end{array}$ & $2.626^{*}$ & 0.010 \\
\hline $\begin{array}{l}\text { Operation time } \\
\left(\mathrm{min} \text {, volume } \leq 160 \mathrm{~cm}^{3}\right)\end{array}$ & $\begin{array}{l}296.4 \pm 36.5 \\
(n=31)\end{array}$ & $\begin{array}{l}310.8 \pm 37.7 \\
(n=28)\end{array}$ & $1.434^{*}$ & 0.157 \\
\hline Cystectomy time (min) & $65.6 \pm 14.0$ & $64.8 \pm 8.7$ & $0.375^{*}$ & 0.708 \\
\hline $\mathrm{EBL}(\mathrm{ml})$ & $550.4 \pm 99.9$ & $477.8 \pm 97.4$ & $4.458^{*}$ & $<0.001$ \\
\hline $\mathrm{Hb}(\mathrm{g} / \mathrm{L})$ & $98.1 \pm 12.5$ & $102.1 \pm 11.6$ & $1.993^{*}$ & 0.048 \\
\hline Transfusion needed & $21(25.0 \%)$ & $13(27.8 \%)$ & 4.277 & 0.039 \\
\hline Time to liquid intake (day) & $1.9 \pm 0.6$ & $2.0 \pm 0.7$ & 1.167 & 0.245 \\
\hline Time to exsufflation (day) & $3.2 \pm 0.6$ & $3.4 \pm 0.8$ & 1.516 & 0.131 \\
\hline Hospital stay after surgery (day) & $7.7 \pm 1.5$ & $7.5 \pm 1.7$ & 0.733 & 0.465 \\
\hline Conversions to open procedure & 1 & 0 & 1.170 & 0.279 \\
\hline
\end{tabular}


Table 3 Cystectomy time based on BMI

\begin{tabular}{lllll}
\hline $\mathrm{BMI}$ & $\mathrm{PLRC}(\mathrm{min}, n)$ & $\mathrm{ALRC}(\mathrm{min}, n)$ & $t$ & $p$ value \\
\hline $\mathrm{BMI} \leq 18.5$ & $52.5 \pm 6.5(12)$ & $60.3 \pm 6.2(16)$ & 3.216 & 0.004 \\
$18.5<\mathrm{BMI} \leq 24$ & $64.7 \pm 11.3(40)$ & $65.2 \pm 8.3(44)$ & 0.223 & 0.825 \\
$\mathrm{BMI}>24$ & $77.4 \pm 14.9(16)$ & $67.8 \pm 10.1(19)$ & 2.264 & 0.030 \\
\hline
\end{tabular}

considered the most common early postoperative complication, and there existed no significant difference between the PLRC and ALRC group. Also, there were no significant differences between the two groups in the incidence of other early complications, including deep vein thrombosis, pyelonephritis, infection, obturator nerve paresis, and wound dehiscence. Likewise, the incidence of late complications including ileus, ureteral stricture, and incisional hernia was comparable.

\section{Discussion}

With the rapid development of laparoscopic techniques, LRC has become an alternative procedure rather than the open radical cystectomy [13]. Besides, LPC is associated with less blood loss and shorter hospital stay and no significant differences in oncologic outcomes [6-8]. The procedure of LPC is complicated, time-consuming, difficult to grasp, and challenging to laparoscopy-naive surgeons $[14,15]$. A large number of studies focused on the technique improvement of LRC, such as the modifications about pelvic lymph node dissection or urinary diversion [16, 17]. The difficulty of laparoscopic cystectomy would increase when working space and vision field were very limited in the pelvis. The present study was to assess the technical modification of laparoscopic cystectomy to make laparoscopic visualization and working space larger.

Compared with female, the pelvis of male is relatively narrower and longer [18], and the skeleton of Asians are generally smaller than the Westerns [19]. These make it even more difficult for surgeons to perform LRC in Asian male patients. In particular, the seminal

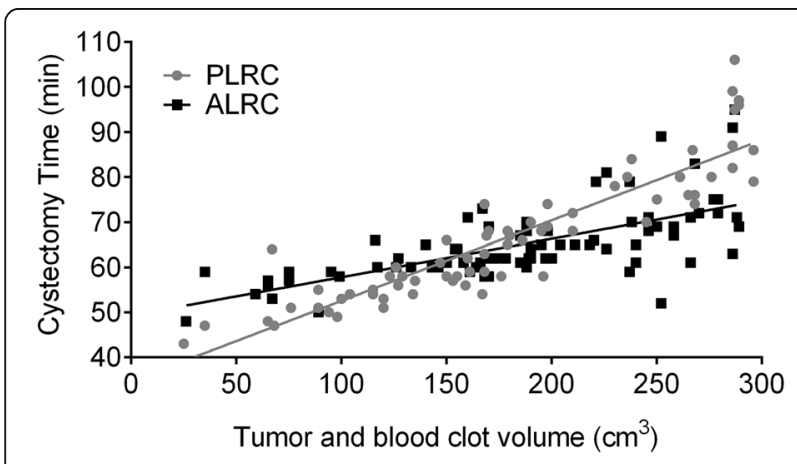

Fig. 2 The linear regression analysis between cystectomy and total tumor and blood clot volume
Table 4 Perioperative complications

\begin{tabular}{|c|c|c|c|c|}
\hline Complications & $\operatorname{PLRC}(n=68)$ & $\operatorname{ALRC}(n=79)$ & $x^{2}$ & $p$ value \\
\hline \multicolumn{5}{|l|}{ Intraoperative } \\
\hline Rectal injury & $2(2.9 \%)$ & $0(0.0 \%)$ & 2.356 & 0.125 \\
\hline External iliac vein injury & $1(1.5 \%)$ & $1(1.3 \%)$ & 0.011 & 0.915 \\
\hline \multicolumn{5}{|c|}{ Early complications ( $\leq 90$ days) } \\
\hline lleus & $2(2.9 \%)$ & $3(4.0 \%)$ & 0.082 & 0.775 \\
\hline Deep vein thrombosis & $2(2.9 \%)$ & $2(2.5 \%)$ & 0.023 & 0.879 \\
\hline Pyelonephritis & $1(1.5 \%)$ & $2(2.5 \%)$ & 0.206 & 0.650 \\
\hline Infection & $1(1.5 \%)$ & $1(1.3 \%)$ & 0.011 & 0.915 \\
\hline Obturator nerve paresis & $1(0.0 \%)$ & $0(0.0 \%)$ & 1.170 & 0.279 \\
\hline Wound dehiscence & $1(1.5 \%)$ & $0(0.0 \%)$ & 1.170 & 0.279 \\
\hline \multicolumn{5}{|l|}{ Late complications (> 90 days) } \\
\hline lleus & $2(2.9 \%)$ & $1(1.3 \%)$ & 0.513 & 0.474 \\
\hline Ureteral stricture & $1(1.5 \%)$ & $1(1.3 \%)$ & 0.011 & 0.915 \\
\hline Incisional hernia & $1(1.5 \%)$ & $0(0.0 \%)$ & 1.170 & 0.279 \\
\hline
\end{tabular}

vesicle and the posterior surface of the prostate become very difficult due to limited vision and working space. To expose the vesicorectal fossa, the posterior plane was developed distally towards the prostate first, usually with the assistant's devices, pull the bladder upwards, and stretch the sigmoid colon towards the head. Besides, a catheter is indwelled routinely preoperative to keep the bladder empty to make the working space largest. For Asian males especially those with large tumor and blood clot, however, these mentioned methods would be still insufficient. The bladder would maintain a certain degree of filling state, and it was hard to produce adequate working place and make the cystectomy more difficult.

In the ALRC procedure, the anterior plane was developed first distally towards the prostate. Then, the bladder was suspended using a 2-0 polyamide stitch towards the abdominal wall (Fig. 1), to reveal the vesicorectal fossa. Even in the cases with large tumor and blood clot, the visualization and working place were excellent. Therefore, the Denonvilliers' fascia was easily encountered at the level of the prostate-vesicular junction, and this fascia can be incised under direct vision, allowing the posterior plane to be developed further distally in a safer way, compared with PLRC. The rectum was less likely to be injured when dissecting the tissue between the prostate and the anterior rectal surface under direct vision. Moreover, for those younger male patients wanted to retain erectile function, laparoscopic nerve-sparing radical cystectomy had been performed with the same principles of open procedure, using ultrasound scalpel to dissect tissue within the prostate fascia to preserve these nerve fibers. 
In the ALRC group, bladder suspension technique provided better surgical exposure and clearer anatomical landmarks, which resulted in much more smooth operation procedures. According to the results, though the average cystectomy time was similar for all patients between the two groups $(64.8 \pm 8.7$ vs $65.6 \pm 14.0)$, the ALRC group required less time for cystectomy when patients' BMI was greater than $24(67.8 \pm 10.1$ vs $77.4 \pm$ 14.9). Also, when total tumor and blood clot volume was larger than $160 \mathrm{~cm}^{3}$, it took less time for the ALRC group (Fig. 2). EBL of the ALRC group was significantly lower than that of the PLRC group $(477.8 \pm 97.4$ vs $550.4 \pm 99.9)$. Better visualization and larger working place played important roles regarding the blood loss. Besides, rectal injury was a common complication and it happened primarily due to the bloody working field between the prostate and the anterior rectal surface. Bladder suspension helped to expose vesicorectal fossa fully and avoid rectal injury. No rectal injury was found here in the ALRC group (Table 4), while there were two cases in the PLRC group.

LRC should be limited to experienced and skilled laparoscopic urologists. The working space in the pelvis and proper laparoscopic visualization are vital for LRC. The bladder lies deeply in the pelvis with a limited space. For female patients, the operative field may be fully revealed by adjusting the surgical posture to a hyperextension lithotomy position, while it remains hard to achieve good better visualization for male patients. In the meantime, there was no effective method reported in the literature to achieve better visualization except the traction of the assistants' devices. Here, a safe and effective alternative technique was reported in detail to achieve better visualization in laparoscopic cystectomy for male patients.

\section{Conclusion}

To sum up, the technique of bladder suspension applied in ALRC is simple and effective to provide better visualization and larger working space. Compared with PLRC, it takes less time of laparoscopic cystectomy for patients with greater BMI or larger total tumor and blood clot volume, and patients also have less blood loss. The technique of bladder suspension is verified to be suitable for Asian male $\mathrm{BCa}$ patients, especially those with greater BMI or larger tumor and blood clot within the bladder.

\section{Acknowledgements}

We thank LetPub (www.letpub.com) for its linguistic assistance during the preparation of this manuscript.

\section{Funding}

This work was supported by National Natural Science Foundation of Hunan (2017JJ3487).
Availability of data and materials

The data sets used or analyzed in this study are available from the corresponding author on reasonable request.

\section{Author's contributions}

$M C$ and LQ designed the study. ST and ZY wrote the article, analyzed the data, and produced the figures and tables. ST, ZY, XZ, YuL, WH, and YaL collected the data. XH and CZ helped in the acquisition of the data. MC, LQ, and $\mathrm{XZ}$ helped the other authors write the revised version. All authors read and approved the final manuscript.

\section{Ethics approval and consent to participate}

The study was approved by the Ethics Committee of Xiangya Hospital, Central South University. Informed consent was obtained from all individual participants included in the study.

\section{Consent for publication}

Informed consent was obtained from the patients for publication of this paper and any accompanying images.

\section{Competing interests}

The authors declare that they have no competing interests.

\section{Publisher's Note}

Springer Nature remains neutral with regard to jurisdictional claims in published maps and institutional affiliations.

Received: 1 November 2018 Accepted: 23 December 2018 Published online: 07 January 2019

\section{References}

1. Antoni S, Ferlay J, Soerjomataram I, et al. Bladder cancer incidence and mortality: a global overview and recent trends. Eur Urol. 2017;71(1):96-108.

2. Burger $M$, Catto JW, Dalbagni $G$, et al. Epidemiology and risk factors of urothelial bladder cancer. Eur Urol. 2013;63(2):234-41.

3. Siegel RL, Miller KD, Jemal A. Cancer statistics, 2018. CA Cancer J Clin. 2018; 68(1):7-30.

4. Li MY, Yang YL, Liu L, et al. Effects of social support, hope and resilience on quality of life among Chinese bladder cancer patients: a cross-sectional study. Health Qual Life Outcomes. 2016;14:73.

5. Alfred Witjes J, Lebret T, Comperat EM, et al. Updated 2016 EAU guidelines on muscle-invasive and metastatic bladder cancer. Eur Urol. 2017;71(3):462-75.

6. Soria F, Moschini M, D'Andrea D, et al. Comparative effectiveness in perioperative outcomes of robotic versus open radical cystectomy: results from a multicenter contemporary retrospective cohort study. Eur Urol Focus. 2018. https://www.doi.org/10.1016/j.euf.2018.11.002.

7. Sathianathen NJ, Kalapara A, Frydenberg M, et al. Robotic-assisted radical cystectomy vs open radical cystectomy: systematic review and metaanalysis. J Urol. 2018. https://www.doi.org/10.1016/j.juro.2018.10.006

8. Tang JQ, Zhao Z, Liang Y, et al. Robotic-assisted versus open radical cystectomy in bladder cancer: a meta-analysis of four randomized controlled trails. Int J Med Robot. 2018;14(1). https://doi.org/10.1002/rcs.1867.

9. Gospodarowicz MK, Brierley JD, Wittekind C. TNM classification of malignant tumours: John Wiley \& Sons; 2017.

10. Froehner M, Brausi MA, Herr HW, et al. Complications following radical cystectomy for bladder cancer in the elderly. Eur Urol. 2009;56(3):443-54.

11. Wein AJ, Kavoussi LR, Novick AC, et al. Campbell-Walsh urology. Elsevier Health Sciences. 2011.

12. Simone G, Papalia R, Ferriero M, et al. Stage-specific impact of extended versus standard pelvic lymph node dissection in radical cystectomy. Int J Urol. 2013;20(4):390-7.

13. Tang K, Li H, Xia D, et al. Laparoscopic versus open radical cystectomy in bladder cancer: a systematic review and meta-analysis of comparative studies. PLoS One. 2014;9(5):e95667.

14. Guo W, Gao L, Zhu M, et al. Application of laparoscopy in urology: a study on the learning curve in experience at a large center including 4707 cases. Int J Clin Exp Med. 2016;9(2):2139-47.

15. Martina GR, Giumelli P, Scuzzarella S, et al. Laparoscopic extraperitoneal radical prostatectomy--learning curve of a laparoscopy-naive urologist in a community hospital. Urology. 2005;65(5):959-63. 
16. Hemal AK, Kolla SB, Wadhwa P, et al. Laparoscopic radical cystectomy and extracorporeal urinary diversion: a single center experience of 48 cases with three years of follow-up. Urology. 2008;71(1):41-6.

17. Kaouk JH, Goel RK, White MA, et al. Laparoendoscopic single-site radical cystectomy and pelvic lymph node dissection: initial experience and 2-year follow-up. Urology. 2010;76(4):857-61.

18. Arsuaga JL, Carretero JM. Multivariate analysis of the sexual dimorphism of the hip bone in a modern human population and in early hominids. Am J Phys Anthropol. 1994;93(2):241-57.

19. Zengin A, Pye SR, Cook MJ, et al. Ethnic differences in bone geometry between White, Black and South Asian men in the UK. Bone. 2016;91:180-5.

Ready to submit your research? Choose BMC and benefit from:

- fast, convenient online submission

- thorough peer review by experienced researchers in your field

- rapid publication on acceptance

- support for research data, including large and complex data types

- gold Open Access which fosters wider collaboration and increased citations

- maximum visibility for your research: over $100 \mathrm{M}$ website views per year

At BMC, research is always in progress.

Learn more biomedcentral.com/submissions 